SLAC-PUB-9207

April 2002

\title{
Plasma Lens Backgrounds at a Future Linear Collider
}

\author{
Achim W. Weidemann \\ Department of Physics, \\ University of South Carolina, \\ Columbia, SC 29208 \\ Pisin Chen, Cho-Kuen Ng \\ Stanford Linear Accelerator Center \\ Stanford, CA 94309
}

\begin{abstract}
A 'plasma lens' might be used to enhance the luminosity of future linear colliders. However, its utility for this purpose depends largely on the potential backgrounds that may be induced by the insertion of such a device in the interaction region of the detector.In this note we identify different sources of such backgrounds, calculate their event rates from the elementary interaction processes, and evaluate their effects on the major parts of a hypothetical Next Linear Collider (NLC) detector. For plasma lens parameters which give a factor of seven enhancement of the luminosity, and using the NLC design for beam parameters as a reference, we find that the background yields are fairly high, and require further study and improvements in detector technology to avoid their impact.
\end{abstract}

Contributed to the 4 th International Workshop,

Electron-Electron Interactions at TeV Energies

Santa Cruz, California, December 7- 9, 2001

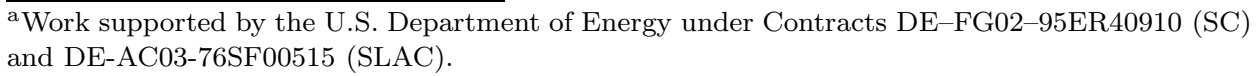




\title{
PLASMA LENS BACKGROUNDS AT A FUTURE LINEAR COLLIDER ${ }^{\dagger}$
}

\author{
ACHIM W. WEIDEMANN ${ }^{\ddagger}$ \\ Department of Physics, University of South Carolina, \\ Columbia, SC 29208 \\ P. CHEN and C.-K. NG \\ Stanford Linear Accelerator Center \\ Stanford, CA 94309
}

\begin{abstract}
A 'plasma lens' might be used to enhance the luminosity of future linear colliders. However, its utility for this purpose depends largely on the potential backgrounds that may be induced by the insertion of such a device in the interaction region of the detector.In this note we identify different sources of such backgrounds, calculate their event rates from the elementary interaction processes, and evaluate their effects on the major parts of a hypothetical Next Linear Collider (NLC) detector. For plasma lens parameters which give a factor of seven enhancement of the luminosity, and using the NLC design for beam parameters as a reference, we find that the background yields are fairly high, and require further study and improvements in detector technology to avoid their impact.
\end{abstract}

Keywords: Linear Collider; Plasma Lens; Backgrounds.

\section{Introduction}

The use of a plasma lens as a final-focusing device to increase the luminosity in $e^{+} e^{-}$ linear colliders was proposed some time ago 1 . Conventional quadrupole magnets for final on-line focusing in high-energy accelerators have limited focusing strengths (a few hundred $\mathrm{MG} / \mathrm{cm}$ ), while plasma lenses are able to produce focusing strengths a few orders of magnitude higher, depending on the plasma density. A plasma-lens, final-focus system might be even more important to achieve a reasonable luminosity in a proposed $e^{-} e^{-}$collider.

Although the self-focusing effect of a plasma lens has been demonstrated experimentally, most recently in experiments at the Final Focus Test Beam at SLAC 2 , the implementation of a plasma lens near a particle detector in a collider requires that the presence of the plasma will not introduce serious backgrounds in the detector as a consequence of beam-plasma interactions. In this note we identify different sources of such backgrounds, calculate their event rates from the elementary interaction processes, and evaluate their effects on the major parts of a hypothetical

${ }^{\dagger}$ Work supported by the U.S. Department of Energy under Contracts DE-FG02-95ER40910 (SC) and DE-AC03-76SF00515 (SLAC).

${ }^{\ddagger}$ Mail Address: SLAC, MS 94, P.O.B. 20450, Stanford, CA 94309. 
Next Linear Collider (NLC) detector.

For plasma lens parameters which may give a factor seven enhancement of the luminosity, and using the NLC design for beam parameters as a reference, we find that the background yields are are fairly high, and require further study and improvements in detector technology or beam time structure to avoid their impact.

\section{Choice of Plasma Lens Parameters}

The background yield depends on both the beam and plasma lens parameters. For the former, we take the NLC beam energy of $E_{\text {beam }}=250 \mathrm{GeV}$ and $N=0.75 \times 10^{10}$ particles per bunch, with $n_{b}=190$ bunches in a bunch train.

For the flat beams of NLC, with r.m.s. beam sizes $\sigma_{x}=245 \mathrm{~nm}, \sigma_{y}=2.7 \mathrm{~nm}$, $\sigma_{z}=110 \mu \mathrm{m}$, 3 it will be difficult to focus further in $y$; however, in the $x$-direction there is some possibility of improvement, as will be shown here.

As an example, we consider then a plasma lens with a density $n_{P}=2 \cdot 10^{18} \mathrm{~cm}^{-3}$, beginning at $s_{0}=1 \mathrm{~cm}$ from the final focus in absence of the lens.

Such a lens would have a focusing strength $K=2 \pi r_{e} n_{P} / \gamma \approx 7.24 \mathrm{~cm}^{-2}$. At the entrance to the lens, the NLC beta functions are changed from their value at the focus in absence of a lens, $\beta_{x 0}^{*}=8 \mathrm{~mm}, \beta_{y 0}^{*}=100 \mu \mathrm{m}$, to

$$
\beta_{x 0}=\beta_{x 0}^{*}\left(1+\left(\frac{s_{0}}{\beta_{x 0}^{*}}\right)^{2}\right)=2.05 \mathrm{~cm},
$$

and similarly, $\beta_{y 0}=100.01 \mathrm{~cm}$.

The differential equation for the $\beta$-function in the plasma lens as a function of the distance $s$ along the beam line, $\beta^{\prime \prime \prime}+4 K \beta^{\prime}+K^{\prime} \beta=0$, can be solved with the initial conditions (matching $\beta, \beta^{\prime}$ at $s=s_{0}$, the entrance to the plasma lens), yielding

$$
\beta=\frac{\beta_{0}}{2}+\frac{1}{2 K \beta_{0}^{*}}+\left(\frac{\beta_{0}}{2}-\frac{1}{2 K \beta_{0}^{*}}\right) \cos \left[\nu\left(s-s_{0}\right)\right]-\frac{2 s_{0}}{\nu \beta_{0}^{*}} \sin \left[\nu\left(s-s_{0}\right)\right]
$$

where $\nu^{2}=4 K$. We chose the thickness of the lens to be

$$
l=\pi / 2 \nu \approx 0.3 \mathrm{~cm} .
$$

The maximal reduction in $\beta^{*}$ can be found to bet

$$
\frac{\beta^{*}}{\beta_{0}^{*}}=\frac{1}{1+K \beta_{0}^{*}\left(\beta_{0}-\beta_{1}\right)}
$$

where $\beta_{0}, \beta_{1}$ are the $\beta$-function at the entrance and exit of the plasma lens, respectively. With the parameters given, one finds $\beta_{x}^{*} / \beta_{x 0}^{*} \approx 1 / 9$, and $\beta_{y}^{*} / \beta_{y 0}^{*} \approx 1 / 6.8$. The factor by which the luminosity increases, given by the square roots of these ratios of $\beta$-functions, thus can be as high as 7.8 , but may be somewhat less in practice due to astigmatism.

Note that the plasma lens parameters chosen above serve only as an illustration and are not necessarily optimized; however, they motivate the choice of the product 
of plasma density and plasma length, $n_{P} \cdot l=0.6 \cdot 10^{18} \mathrm{~cm}^{-2}$ as conservative for a study of backgrounds produced by a plasma lens at the NLC.

Here we consider only a plasma lens focusing one beam just before the interaction point; having a plasma lens for both beams would, of course, double the backgrounds. One could also imagine having a suitably shaped plasma at the interaction point itself.

\section{Sources of Backgrounds}

Background particles produced by a plasma lens are of three types, namely, electrons/positrons, hadrons, and photons. These particles originate from different elementary physical processes underlying the interactions of the incoming electron or positron beam with the plasma of the lens located near the interaction point. In this section, we outline all the processes responsible for the various sources of backgrounds 0 . Their cross sections and angular distributions are calculated in the next section. From these results and the parameters shown, the number of background particles can be determined and their effect on the detector evaluated.

\subsection{Electrons}

Electrons and positrons arise from the scattering of the $e^{+}$or $e^{-}$beam with the electrons of the plasma. The processes for producing electrons and positrons are then

Bhabha scattering, $e^{+} e^{-} \rightarrow e^{+} e^{-}$,

Møller scattering, $e^{-} e^{-} \rightarrow e^{-} e^{-}$,

elastic scattering, $e p \rightarrow e p$,

and inelastic scattering, $e p \rightarrow e X$.

\subsection{Hadrons}

The hadronic backgrounds come from the elastic and inelastic scattering of the $e^{+}$ or $e^{-}$beam, and also from the inelastic scattering of photons (from synchrotron radiation and bremsstrahlung), with the protons in the plasma. Hadrons are produced by

elastic scattering, $e p \rightarrow e p$,

and inelastic scattering, $e p \rightarrow e X, \gamma p \rightarrow X$.

\subsection{Photons}

Photon backgrounds from a plasma are produced by

Compton scattering, $e \gamma \rightarrow e \gamma$. 
Four mechanisms produce the incident photons: synchrotron radiation at the finalfocus quadrupole, beamstrahlung from the colliding beams at the interaction point, synchrotron radiation as a consequence of plasma focusing, and bremsstrahlung of the $e^{+}$or $e^{-}$beam in the plasma. These photons are then scattered by plasma electrons into large angles.

Note that all detectors at $e^{+} e^{-}$colliders have to cope with photon backgrounds generated by synchrotron radiation and beamstrahlung scattering off masks or similar structures, which is not considered here.

\section{Cross Sections and Event Rates}

The cross section of an electron or a photon scattered by the plasma for a particular process is calculated by integrating its angular distribution

$$
\sigma=\int_{\theta_{c}}^{\pi-\theta_{c}} \frac{d \sigma}{d \Omega} d \Omega
$$

where $\theta_{c}$ is the angular cut for the scattered particles into the detector, which is taken to be $150 \mathrm{mrad}$ in our calculations. The number of scattered particles $N_{s}$ for a bunch train is then given by

$$
N_{s}=n_{b} \mathcal{L} \sigma
$$

where $n_{b}$ is the number of bunches in a bunch train, and $\mathcal{L}$ is the beam-plasma luminosity per bunch crossing, respectively. The beam-plasma luminosity is given by

$$
\mathcal{L}=N n_{P} l,
$$

where $N$ is the number of particles in a bunch, $n_{P}$ is the plasma density and $l$ is the thickness of the plasma.

With the beam and plasma lens parameters of section 2 above, the beam-plasma luminosity $\mathcal{L}$ is then $4.5 \cdot 10^{27} \mathrm{~cm}^{-2}$. For an anticipated NLC luminosity ${ }^{3}$ of $\mathcal{L}_{N L C}=$ $2 \cdot 10^{34} \mathrm{~cm}^{-2} \mathrm{~s}^{-1}$, the beam-beam luminosity per bunch crossing, $\mathcal{L}_{N L C} /\left(120 \mathrm{~Hz} \cdot n_{b}\right)$ is $8.8 \cdot 10^{29} \mathrm{~cm}^{-2}$, two orders of magnitude larger than the beam-plasma luminosity. For a bunch train of $n_{b}=190$ bunches, $n_{b} \mathcal{L}=8.55 \cdot 10^{29} \mathrm{~cm}^{-2}$.

While the energy of the electrons in the beam is monochromatic, the energy of the photons incident on the plasma lens is not. The photons originating from the final-focus quadrupole and the plasma focusing, as well as those from the beamstrahlung at the interaction point, follow the synchrotron-radiation spectrum, while those from bremsstrahlung in the plasma follow the Weizsäcker-Williams spectrum. Thus the photon distribution can be written as

$$
n_{\gamma}(y)=n_{S R}^{p}(y)+n_{S R}^{q}(y)+n_{\text {Brem }}(y)+n_{\text {Beamst }}(y),
$$

where $y=E_{\gamma} / E_{\text {beam }}$ is the ratio of the photon energy to the beam energy, $n_{\gamma}(y)$ is the total photon spectrum, and $n_{S R}^{p}(y), n_{S R}^{q}(y), n_{B r e m}(y)$ and $n_{\text {Beamst }}(y)$ are the 
contributions from plasma-lens focusing, quadrupole focusing, bremsstrahlung and beamstrahlung, respectively. The synchrotron radiation spectrum from a focusing system can be approximated by the following expression 6 :

$$
n_{S R}^{i}(y)=\frac{1}{\pi} \Gamma\left(\frac{2}{3}\right)\left(\frac{\alpha d_{i}}{\sqrt{3} \gamma \lambda_{e}}\right)\left(3 \Upsilon_{i}\right)^{2 / 3} y^{-2 / 3}, \quad 0 \lesssim y \lesssim \Upsilon_{i}, \quad i=p, q, b .
$$

Here $\lambda_{e}$ is the electron-Compton wavelength, $\gamma$ is $E_{\text {Beam }} / m_{e} c^{2}, d_{i}$ is the length of the focusing element $\left(d_{q}=1 \mathrm{~m}\right.$ for a quadrupole and $d_{p}=l=3 \mathrm{~mm}$ for a plasma lens), and $\Upsilon_{i}=2 E_{c}^{i} / 3 E_{\text {beam }}$, where $E_{c}^{i}$ is the synchrotron-radiation critical energy. For $y>\Upsilon_{i}$, the synchrotron-radiation power is exponentially small and is neglected here. For a final-focus quadrupole, $\Upsilon_{q} \sim 1 \cdot 10^{-5}$ and $E_{c}^{q} \sim 2.5 \mathrm{MeV}$; for the plasma lens considered here, $\Upsilon_{p} \sim 0.05$ and $E_{c}^{p} \sim 17.3 \mathrm{GeV}$. The beamstrahlung spectrum, $n_{\text {Beamst }}(y)$, can also be described by (9), that is $n_{\text {Beamst }}(y)=n_{S R}^{b}(y)$, with parameters $3 \Upsilon_{p} \sim 0.11, E_{c}^{p} \sim 41.25 \mathrm{GeV}$ and $d_{q}=2 \sqrt{3} \sigma_{z}=0.38 \mathrm{~mm}$. This formula will overestimate the beamstrahlung spectrum, as the integral over $n_{\text {Beamst }}(y)$ with the upper limit 0.2 yields $\sim 3$, rather than the 1.2 photons per incident electron indicated in the 2001 Report on the NLCl.

The bremsstrahlung spectrum from beam electrons scattered in the plasma is given by the Weizsäcker-Williams spectrum

$$
n_{\text {Brem }}(y)=\frac{2 \alpha}{\pi y}\left[\ln 2.246+\ln \frac{m_{p}}{m_{e}}-\frac{1}{2}-\ln y\right],
$$

where $m_{p}$ and $m_{e}$ are the proton and electron masses, respectively. Then the angular distribution of the cross section for each Compton process, after taking the photon spectrum into account, is given by

$$
\frac{d \sigma}{d \Omega}=\int_{0}^{y_{0}} \frac{d \sigma(e \gamma \rightarrow e \gamma)}{d \Omega} n(y) d y
$$

where $n(y)$ is either the synchrotron radiation or the bremsstrahlung spectrum, and $y_{0}$ is the value above which radiation can be neglected, which is $\sim 1.5 \cdot 10^{-5}, 0.15$ and 1 for quadrupole focusing, plasma focusing, and bremsstrahlung in the plasma, respectively, and 0.2 for beamstrahlung.

The integrated cross sections and backgrounds from the different processes are summarized in Table 1. We now discuss in more detail the production of each type of background particle.

\subsection{Electrons}

The number of particles scattered into the detector depends on the angular acceptance, which is taken to be $150 \mathrm{mrad}$ to $\pi-150 \mathrm{mrad}$ from the incident-beam direction. When a $250-\mathrm{GeV}$ electron or positron hits an electron at rest, the scattered particles go in the forward direction within a cone of very small opening angle about the direction of the incoming electron. Thus we expect a very small number of electrons scattered into the detector when a beam passes through the plasma 
Table 1: Summary of background sources from a plasma lens in NLC for a single beam crossing. The cross sections $\sigma_{\text {tot }}$ are integrated as in Eq. (11) and (5); energy cuts (of $4-100 \mathrm{keV},>100 \mathrm{keV}$ ) were imposed in the calculation of particle numbers in the last two columns; see Section 5.

\begin{tabular}{|c|c|c|c|}
\hline Background source & $\begin{array}{c}\sigma_{\text {tot }}\left(\mathrm{cm}^{-2}\right) \\
|\cos \theta| \leq 0.99\end{array}$ & $\begin{array}{c}\text { Vertex } \\
\text { detector }\end{array}$ & $\begin{array}{c}\text { Drift } \\
\text { chamber }\end{array}$ \\
\hline Bhabha and Møller $e^{+}, e^{-}$ & 0 & 0 & 0 \\
\hline Elastic ep: e & $0.103 \times 10^{-45}$ & negligible & negligible \\
\hline 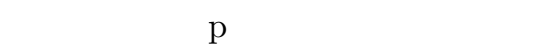 & $0.613 \times 10^{-39}$ & negligible & negligible \\
\hline Inelastic ep: e & $0.132 \times 10^{-33}$ & negligible & negligible \\
\hline charged hadrons & $0.396 \times 10^{-29}$ & 0.021 & 0.021 \\
\hline Inelastic $\gamma p$ : charged hadrons & $0.372 \times 10^{-28}$ & 0.139 & 0.139 \\
\hline Compton $\gamma^{\prime}$ s from quadrupole & $0.18 \times 10^{-24}$ & 270 & 380 \\
\hline Compton $\gamma^{\prime}$ s from plasma focusing & $0.23 \times 10^{-24}$ & 290 & 580 \\
\hline Compton $\gamma^{\prime}$ s from bremsstrahlung & $0.19 \times 10^{-23}$ & 970 & 480 \\
\hline Compton $\gamma$ 's from beamstrahlung & $0.52 \times 10^{-25}$ & 70 & 130 \\
\hline
\end{tabular}

lens. Electron backgrounds are essentially zero for Bhabha and Møller scatterings, since the scattered particles come out within the cone specified by our angular cut.

The angular distributions of the cross sections of the electrons and protons produced by elastic $e p$ scattering are shown in Fig. 1. The cross section for electron production peaks highly in the forward direction. The total cross section for the imposed angular cut is $0.103 \cdot 10^{-45} \mathrm{~cm}^{-2}$, and hence, the contribution to the electron sources is negligibly small.

The angular distribution of the scattered electrons for inelastic ep scattering can be obtained by integrating the following differential distribution $\mathrm{l}$ over energy:

$$
\frac{d \sigma}{d E^{\prime} d \Omega}=\frac{\alpha^{2}}{4 E^{2} \sin ^{4} \frac{\theta}{2}}\left\{W_{2}\left(\nu, q^{2}\right) \cos ^{2} \frac{\theta}{2}+2 W_{1}\left(\nu, q^{2}\right) \sin ^{2} \frac{\theta}{2}\right\} .
$$

Here $E$ and $E^{\prime}$ are the incoming and outgoing electron energies respectively, $\nu=$ $E-E^{\prime}, q^{2}$ is the momentum transfer squared, and $\alpha$ is the fine-structure constant. The parametrizations of the structure functions $W_{1}\left(\nu, q^{2}\right)$ and $W_{2}\left(\nu, q^{2}\right)$ are taken from Ref. . The angular distribution of the cross section for inelastic $e p$ scattering is shown in Fig. 2(a). Again, we see that the cross section peaks highly in the forward direction. The total cross section is $0.132 \cdot 10^{-33} \mathrm{~cm}^{2}$, which gives $0.11 \cdot 10^{-3}$ electrons for a bunch train. Hence it can be concluded that the electron backgrounds due to the presence of a plasma lens can be neglected.

\subsection{Hadrons}

While the electrons essentially move in the forward direction, the protons from $e p$ elastic scattering are scattered into larger angles. In Fig. 1 we see that the 


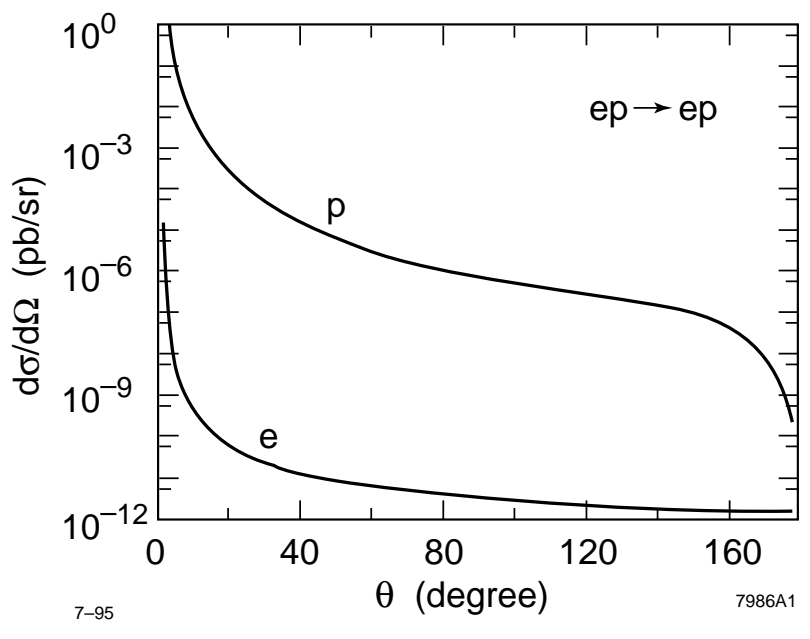

Fig. 1

Fig. 1. Angular distributions of the cross sections in the laboratory frame for ep elastic scattering. The energy of the incoming electron is $250 \mathrm{GeV}$ and the proton is at rest.

differential cross section is a few orders of magnitude bigger than that for the electrons. Nevertheless, the integrated cross section is $0.613 \cdot 10^{-39} \mathrm{~cm}^{2}$ and hence the contribution to the proton sources is negligibly small. For inelastic ep scattering, the initial-state proton can disintegrate into other particles; to properly take this effect into account we use HERWIG 10 to simulate the inelastic ep reactions of 250$\mathrm{GeV}$ electrons with protons at rest. The differential distribution of charged hadrons as a function of scattering angle is shown in Fig. 2(b); it again peaks at small angles. The total cross section for the angular cut of $150 \mathrm{mrad}$ is found to be $0.396 \cdot 10^{-29}$ $\mathrm{cm}^{2}$, which corresponds to about 3.4 charged hadrons for a bunch train at NLC energy. Thus, we expect the hadronic backgrounds from ep scattering will not pose problems to any component of a NLC detector.

For inelastic $\gamma p$ scattering, where the photon originates from one of the mechanisms mentioned in a previous section, we found from HERWIG simulations that the cross section does not vary sensitively with the incoming photon energy. Hence, we considered a monochromatic photon of $10 \mathrm{GeV}$ interacting with a proton at rest for estimating the cross section. The integrated cross section is found to be $0.372 \cdot 10^{-28} \mathrm{~cm}^{2}$, which is about 8 times larger than that of the $e p$ inelastic scattering. This corresponds to 32 charged hadrons for a bunch train, and hence this background is still very small. 


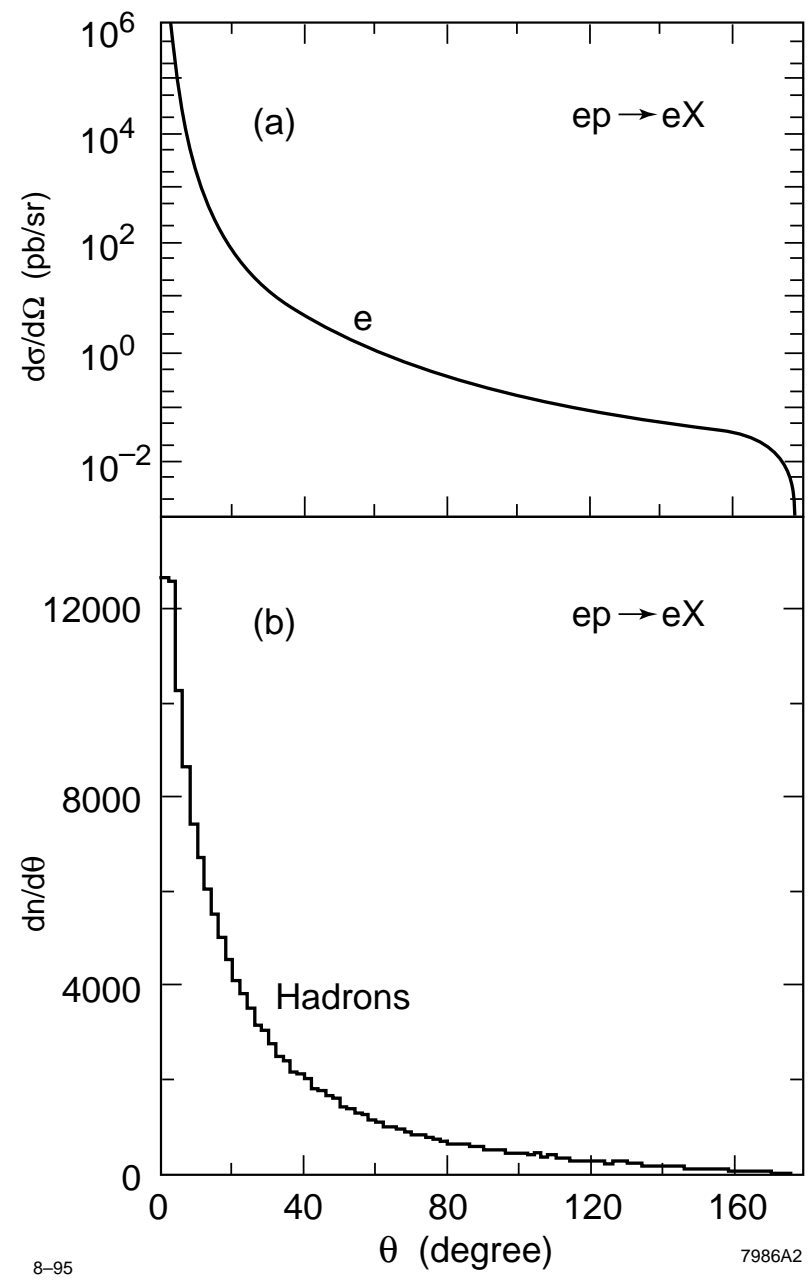

Fig. 2

Fig. 2. (a) Angular distribution of the cross section in the laboratory frame for the scattered electrons in $e p$ inelastic scattering. (b) HERWIG angular distribution of charged hadrons for 20,000 events for $e p$ inelastic scattering. The total cross section is normalized to $1.02 \cdot 10^{7} \mathrm{pb}$.

\subsection{Photons}

The photon backgrounds for the Compton process from the four photon sources depend on the photon spectrum. When the photon energy is much greater than the electron rest mass, the Compton cross section has a peak in the forward direction. When the photon energy is comparable-to or less-than the electron mass, the scattering is reduced to Thomson scattering and the angular dependence of the cross section varies as a dipole distribution. The angular distributions of the 

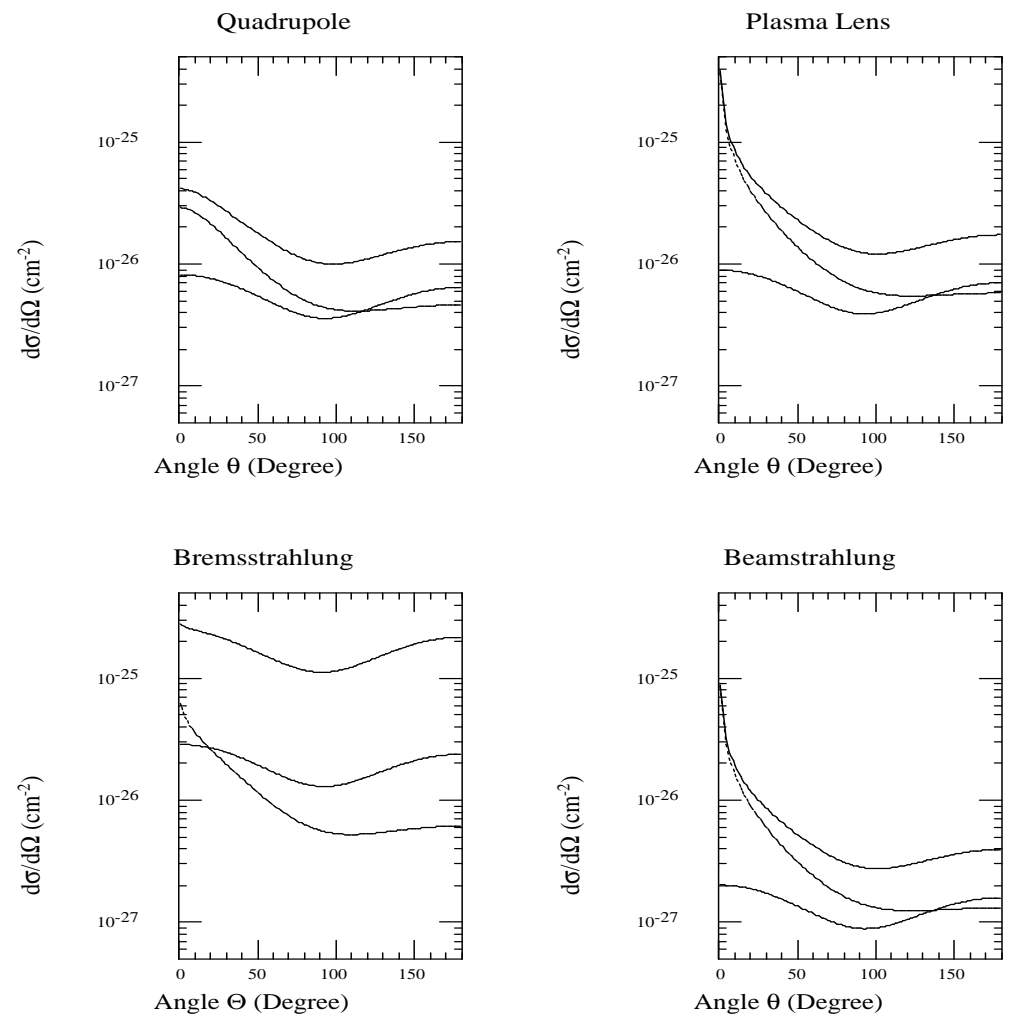

Fig. 3. Angular distributions of the Compton cross sections from the four sources of photons: quadrupole focusing, plasma-lens focusing. bremsstrahlung in plasma,beamstrahlung. The topmost solid lines are integrated over the whole energy range, the next lower ones for the energy range between $4 \mathrm{keV}$ and $100 \mathrm{keV}$ (relevant for the vertex detector), and the lines lowest (at right-hand side of plot) are for photons of energy greater than $100 \mathrm{keV}$ (relevant for the drift chamber).

cross sections of the Compton process from the four photon sources are shown in Fig. 3. The bremsstrahlung spectrum is very soft; the angular distribution of this process is quite flat: it looks like a dipole distribution. The typical energy of the photon for synchrotron radiation from final quadrupole focusing is still small (on the order of one $\mathrm{MeV}$ ) and hence, the distribution does not vary drastically with angle although it peaks moderately in the forward direction. Plasma focusing is much stronger than quadrupole focusing and the photon spectrum is expected to be harder with the typical energy on the order of $10 \mathrm{GeV}$. The cross section peaks in the forward direction and then drops rapidly at large angles. The total number of photons of all energies for a bunch train is $\sim 1,990,000$, or about 10,500 for a single beam crossing. (Photons above $1 \mathrm{keV}$ are $\sim 722,000$ per bunch train, or 3,800 per single beam crossing, still a considerable number.) The photon backgrounds from $\gamma p$ inelastic scattering are in general a few orders of magnitude smaller than 
those from Compton scattering, and hence can be neglected.

\section{Detector Backgrounds}

As discussed in Section 4, the leptons and hadrons scattered into the fiducial volume of a detector can be neglected. The photons created by the different mechanisms calculated above cover a wide range of energy. Different components of future linear collider detectors are sensitive to certain energy windows of the energy spectrum of the outcoming photons. We now consider the photon backgrounds in a vertex detector, a tracking device such as a drift chamber or TPC, and a calorimeter of an idealized NLC detector.

\subsection{Vertex detector}

A description of the general characteristics of a pixel-based vertex detector for the NLC can be found in Ref. 11 .

A minimum-ionizing charged particle will deposit $7.75 \mathrm{keV}$ and generate about 2,100 electron-hole pairs in the, say, $20-\mu \mathrm{m}$-thick active Silicon of a vertex detector. Photons above $50 \mathrm{keV}$, for example, are unlikely to convert in the very thin structure of a barrel-vertex detector, which is expected to have a thickness of as little as $0.11 \%$ of a radiation length 11 . Thus, to be conservative, background photons of energies between, say, $4 \mathrm{keV}$ and $100 \mathrm{keV}$ need to be considered here. With these energy cuts, the number of background photons for the vertex detector is $\sim 303,000$ for a bunch train, or 1600 per single-beam crossing, compared with about $1.99 \cdot 10^{6}$ photons per bunch train at all energies.

The angular distributions of the cross sections from different mechanisms are shown in Fig. 3; The cross section from bremsstrahlung photons is reduced by more than an order of magnitude because of the cut at the lower end of the soft Weizsäcker-Williams spectrum. The cross sections from quadrupole and plasma focusing photons are roughly reduced by a factor of 3 .

For a vertex-detector barrel with a radius of $2.5 \mathrm{~cm}$ and with a length of 33 $\mathrm{cm}$ covering angles greater than $150 \mathrm{mrad}(|\cos \theta| \leq 0.99)$, the density of photon tracks per unit surface area is $\sim 5.8 / \mathrm{mm}^{2}$ for a bunch train, which is more than the tolerable occupancy of $\sim 1 / \mathrm{mm}^{2}$. In comparison, the 'Snowmass 2001' detector design 12 forsees an occupancy of $\sim 1 / \mathrm{mm}^{2}$ per bunch train at a radius of $1.2 \mathrm{~cm}$.

Note that the choice of $|\cos \theta| \leq 0.99$ for the vertex detector is very generous, however the number of background photons could only be slightly reduced if the angular coverage is limited to, for example, $|\cos \theta| \leq 0.90$. (Then there are still $\sim 264,000$ photons per bunch train, $\sim 1,400$ per bunch crossing). A better method would be to change the beam time structure, that a vertex detector does not integrate noise over 190 pulses; and to increase the readout speed of the device. The noise integrated only over, say 30 bunches, would lead to an average occupancy of $\sim 0.9 / \mathrm{mm}^{2}$. 


\subsection{Tracking}

The next layer in the detector may well be a large drift chamber or TPC with about 10,000 sense wires. Such a drift chamber can easily tolerate 100 random background hits (1\% occupancy). Assuming a 1\% conversion probability, this allows for 10,000 incident photons. Photons of very small energies, say $\lesssim 10 \mathrm{keV}$, will be absorbed by the beam pipe and can be ignored. The conversion products of photons of less than, say $100 \mathrm{keV}$, will not form track segments. In a typical magnetic field they will form tight loops and deposit their energy locally. Proper design of the readout should account not only for the charge distribution typical of minimum-ionizing particles, but also for the sometimes large local depositions of energy from converting photons. Thus we need only to consider photons of energy greater than $100 \mathrm{keV}$ scattered into a drift chamber or other tracking system.

The angular distributions of the cross sections above $100 \mathrm{keV}$ are also shown in Fig. 3. They all peak in the forward direction as a result of the hard-photon spectra from all the mechanisms. The total number of background photons with energy above $100 \mathrm{keV}$ and scattering angle greater than $150 \mathrm{mrad}$ is about 299,000 per bunch train, or $\sim 1,600$ per beam crossing. Notice that we integrated the background over a whole bunch train. Again, increasing the angular cut to $451 \mathrm{mr}$ or $|\cos \theta| \leq$ 0.90 leads only to modest improvement ( $\sim 240,000$ photons per bunch train, $\sim 1,300$ per bunch crossing). By the time NLC is built, we expect that progress in tracking technology (e.g. 'bunch taggging 12 ) will allow a better separation of tracks from adjacent bunch crossings, such that only the background from a few adjacent bunch crossings (instead of 190) needs to be considered.

\subsection{Calorimeter}

As mentioned above, leptons and hadrons will not be scattered into the main part of a detector in sufficient numbers to cause a problem for the calorimeter of a hypothetical NLC detector. Photons of very low energies will have been absorbed by the beampipe, drift-chamber walls, and similar structures before reaching the calorimeter. A typical calorimeter can possibly see energies as low as a few $\mathrm{MeV}$; however, for any actual analysis, a cut is made removing clusters in a calorimeter with a total energy below, say, $100 \mathrm{MeV}$. Our calculation shows that of the total flux per bunch train of about $1.99 \cdot 10^{6}$ photons of all energies in the angular region with $|\cos \theta| \leq 0.99,5,950$ have an energy above $100 \mathrm{MeV}$,(i.e. 31 per beam crossing,) and about 109,000 per bunch train, or $\sim 570$ per single-beam crossing, have an energy between 1 and $100 \mathrm{MeV}$. While some of the latter might be seen in the innermost layer of electromagnetic calorimetry, they do not present a large problem and are easily removed by a cluster cut, if isolated. The energy added by them to a shower created by a high-energy particle or jet is negligible compared to the intrinsic resolution of a typical calorimeter. Removal of the $\sim 31$ higher-energy clusters per beam crossing requires more thought. Due to the higher energy of the relevant photons, a larger angular cut helps more and removes about $40 \%$ of these 
photons; in the region $|\cos \theta| \leq 0.90$, about 3,500 photons per bunch train or 18 per bunch crossing remain with energy above $100 \mathrm{MeV}$.

Calorimeter backgrounds might also be attributed to individual bunch crossings by the use of scintillation counters or similar fast timing devices, so that again integration of the backgrounds over a bunch train is excessively conservative.

\section{Summary}

We summarize our background calculations in Table 1, which shows the various cross sections and particle fluxes for a single bunch crossing from the different background sources. using NLC parameters as a reference. We have estimated only the sources of backgrounds from a plasma lens for a generic particle detector, but did not consider here at all the sources of backgrounds generated by collisions without a plasma lens that are common to any NLC detector, such as those due to beam scrapings or synchrotron radiation scattering off masks or similar structures, or the direct beamstrahlung from the opposing beam. (The compton scattering of the beamstrahlung on the plasma lens was included.)

We have been rather conservative in our assumptions of the plasma lens parameters; presumably one with half the density (and thus producing half as much backgrounds), if properly optimized, might still increase the luminosity by a factor of close to five.

Moreover, we have further assumed that the resolution time for the detector is much longer than $1.4 \mathrm{~ns}$, the separation between successive bunches in a bunch train, but less than the $\sim 8.3 \mathrm{~ms}$ separation between bunch trains. and have integrated in our discussion the backgrounds over a train of 190 bunches. for a total time span of $\sim 270 \mathrm{~ns}$. The thus-integrated backgrounds, overlayed over those produced by collisions without a plasma lens, present indeed a challenge, and require further study by a bona fide simulation of both beams, plasma lens, and detectors. Improved, especially faster, detector technology, may be available by the time a future high-energy linear collider is built; this, together with an optimized design (perhaps allowing for lesser plasma density and a different time structure of the beam) will possibly make the additional backgrounds created by a plasma lens more acceptable, and as amenable as those anyway created by high-energy collisions. Thus, the use of a plasma lens should certainly be considered carefully for the case that the conventional focusing should not give the desired results, or to increase the luminosity for certain physics channels which might be easier separated from the backgrounds.

\section{Acknowledgements}

We thank Clem Heusch for organizing again a stimulating workshop, and T. Barklow, C.J.S. Damerell, S. Hertzbach, T. Markiewicz, S. Rajagopalan, D. Su, and E. Vella for useful discussions. 
14 A.W.Weidemann, P.Chen, C.-K.Ng

\section{References}

1. P. Chen, Part. Accel. 17, 121 (1987).

2. J.S.T. Ng, et al. (E-150 Collaboration), Phys.Rev.Lett. 87, 244801 (2001).

3. The NLC Collaboration, 2001 Report on the Next Linear Collider, N. Phinney,editor, FERMILAB-Conf-01/075-E, SLAC-R-571, June 2001, p.20.

4. P. Chen, S. Rajagopalan, J Rosenzweig, Phys. Rev. D40, 923 (1989).

5. C. Baltay, Backgrounds in SLD due to the Proposed SLC Plasma Lens, SLD Internal Note, 1992 (unpublished).

6. P. Chen, Beamstrahlung and the QED, QCD Backgrounds in Linear Colliders, SLACPUB-5914, 1992; and in Photon-Photon Collisions, D. O. Caldwell and H. P. Paar, ed., World Scientific (1992).

7. See, for example, J. D. Jackson, Classical Electrodynamics, 2nd ed. John Wiley \& Sons (1975), p.724.

8. See, for example, F. Halzen and A. D. Martin, Quarks and Leptons, New York, Wiley (1984), p.179.

9. J. Kwiecinski, A. D. Martin, R. G. Roberts and W. J. Stirling, Phys. Rev. D42, 3645 (1990).

10. G. Marchesini et al., HERWIG: A Monte Carlo Event Generator for Simulating Hadron Emission Reactions with Interfering Gluons, Comp. Phys. Comm. 67, 465 (1992).

11. C. J. S. Damerell, Vertex Detectors at Linear Colliders (Present and Future), RAL Report RAL-94-096, 1994.

12. The American Linear Collider Working Group, Linear Collider Physics - Resource Book for Snowmass 2001, FERMILAB-Pub-01/058-E, SLAC-R-570, June 2001, p.386. 